\title{
HIGHLIGHTS
}

POLYCYSTIC KIDNEY DISEASE

\section{Renal transplantation in patients with ADPKD - the good, the bad and ugly}

A large study offers fresh insight into the long-term outcomes of kidney transplantation in patients with autosomal dominant polycystic kidney disease (ADPKD). "Renal transplantation in patients with ADPKD is associated with better graft survival [but] more thromboembolic complications, more metabolic complications and increased rates of hypertension..." write Jacquet and colleagues in Transplant International.

The researchers reviewed data on 534 patients with ADPKD and 4,779 patients with renal failure unrelated to ADPKD (controls). The patients were followed up for $\leq 15$ years (median 7 years). Validated data for all patients were prospectively entered into an electronic database that included donor and recipient baseline characteristics, clinical records and the causes of graft failure and patient death.

"The subjective clinical feeling was that patients with ADPKD face specific infectious problems," comments Nicolas Pallet, corresponding author of this multicenter French study. However, this expectation proved to be unfounded: the prevalence of bacterial infection was similar in both groups. Interestingly, the improved graft survival observed in patients with ADPKD occurred despite an increased incidence of several donorrelated and procedure-related risk factors for graft rejection. The researchers did, however, find increased rates of metabolic complications (including new-onset diabetes after transplantation) and hypertension, and an increased risk of thromboembolism in patients with ADPKD; the latter association has not previously been reported. "Our data emphasize that transplanted ADPKD patients would benefit from venous thromboembolism prevention," concludes Jacquet et al's article. Pallet also calls for radiographic and coagulation

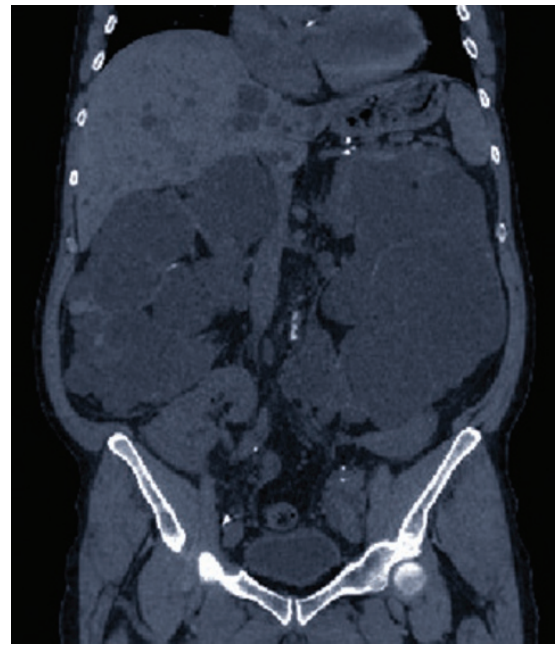

Coronal CT image: hepatorenal cysts and a kidney allograft in a patient with ADPKD. Image courtesy of Nicolas Pallet, Necker Hospital, Paris, France.

studies to improve understanding of the thromboembolic complications in patients with ADPKD.

Steven E. Bradshaw

Original article Jacquet, A. et al. Outcomes of renal transplantation in patients with autosomal dominant polycystic kidney disease: a nationwide longitudinal study. Transpl. Int. doi:10.1111/j.1432-2277.2011.01237.x 